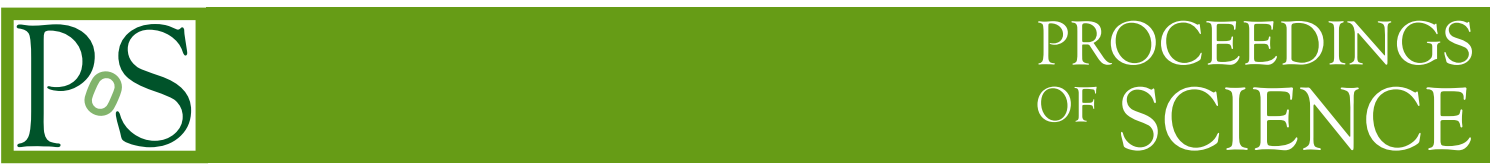

\title{
Flow observables and the nuclear equation of state
}

\author{
Wolfgang TRAUTMANN* \\ GSI Helmholtzzentrum Gmbh, D-64291 Darmstadt, Germany \\ E-mail: w.trautmann@gsi.de
}

Collective flows in heavy-ion reactions at intermediate energies have been shown to be useful observables for investigating the reaction dynamics and for determining the parameters of reaction models based on transport theory. More recently, the elliptic flow in collisions of neutron-rich heavy-ion systems has been found to be particularly sensitive to the strength of the symmetry energy and to its dependence on density near and above saturation. The comparison of ratios or differences of neutron and charged-particle flows with predictions of transport models favors an approximately linear density dependence of the symmetry energy.

The degree of potential model dependences of these results is a topic of high current interest. Extensive parameter searches using different versions of QMD transport models have shown that the overall dependence on model parameters is comparable to the uncertainties of existing experimental data. Constraining the parameters used in transport theory will thus be as important as comprehensive new flow data of high accuracy in order to improve our knowledge of the equation of state of asymmetric nuclear matter.

52th International Winter Meeting on Nuclear Physics (Bormio 2014)

27-31 January 2014

Bormio, Italy

${ }^{*}$ Speaker. 


\section{Introduction}

The rich information contained in flow observables is well documented in the comprehensive account of the measurements of the FOPI Collaboration presented recently in "Systematics of azimuthal asymmetries in heavy ion collisions in the $1 \mathrm{~A} \mathrm{GeV}$ regime" [1]. As an example from this work, results for the observed directed and elliptic flows of deuterons from mid-central ${ }^{197} \mathrm{Au}$ $+{ }^{197} \mathrm{Au}$ collisions at $1.5 \mathrm{GeV}$ per nucleon incident energy are shown in Fig. 1. Even if the reaction, beam energy, impact parameter, and particle type are specified, collective flows are still functions of transverse momentum and rapidity. These latter dependences are shown in the present example for the indicated intervals of the respectively other quantity. The variables used here are the transverse component $u_{t}=\beta_{t} \gamma$ of the four-velocity $u=(\gamma, \beta \gamma)$, normalized with respect to the velocity $u_{p}$ of the incident projectile in the center-of-mass (c.m.) system, i. e. $u_{t 0} \equiv u_{t} / u_{p}$, and the reduced c.m. rapidity $y_{0} \equiv y / y_{p}$, normalized with respect to the c.m. projectile rapidity $y_{p}$.
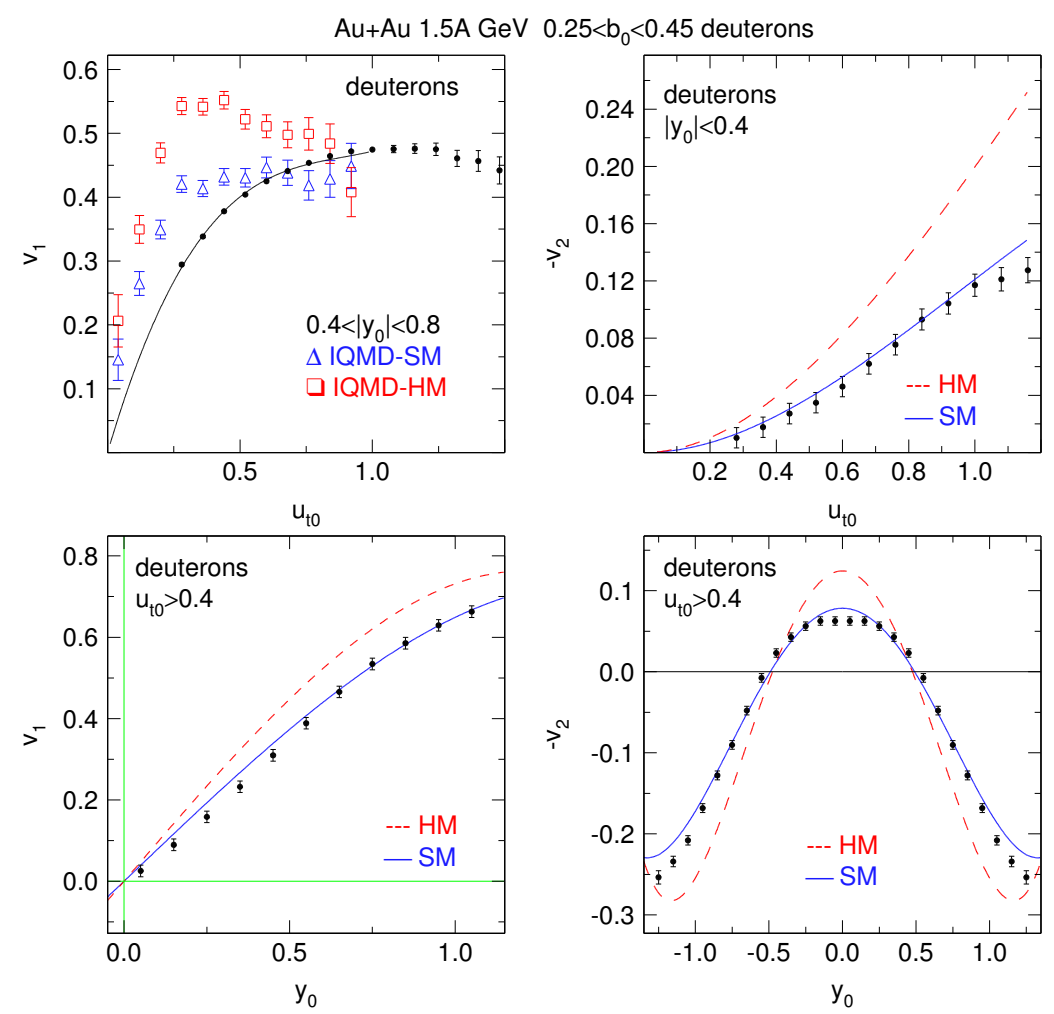

Figure 1: Directed (left panels) and elliptic (right panels) flows of deuterons in ${ }^{197} \mathrm{Au}+{ }^{197} \mathrm{Au}$ collisions at $1.5 \mathrm{GeV}$ per nucleon incident energy and centrality $0.25<b_{0}<0.45$. The dependences on the transverse velocity $u_{t 0}$ and rapidity $y_{0}$ (see text) for the indicated rapidity and transverse-velocity intervals are shown in the upper and lower panels, respectively, together with IQMD calculations (lines) using a soft (SM) and a hard (HM) equation of state with momentum dependence. Note the inversion of $v_{2}$ and the definition of the reduced impact parameter $b_{0}=b / b_{\max }$ (reprinted from Ref. [1], Copyright (2012), with permission from Elsevier).

The experimental results are compared with predictions of the isospin-dependent quantummolecular dynamics (IQMD) transport model in the version of Ref. [2]. What is most impressive 
is not only the precise agreement with the measurements if the choice of the soft equation of state (EoS) is made but perhaps even more the fact that, as shown in the paper, the agreement of the illustrated kind extends over the covered range of incident energies from $400 \mathrm{MeV}$ per nucleon to $1.5 \mathrm{GeV}$ per nucleon and for protons, deuterons and tritons, all with the same model assumptions. The variables $v_{1}$ and $v_{2}$ describing directed and elliptic flows (see below for their definitions) are well reproduced. The agreement is not perfect everywhere, as evident from the upper left panel of the figure presenting the transverse-velocity dependence of the directed deuteron flow in the indicated intermediate rapidity interval. Small deviations occur also in the description of the elliptic flow at the extreme transverse velocities and rapidities (Fig. 1, right panels). It is evident, however, that differential measurements over a wide range of the variable space are indispensable for reaching convincing conclusions, here the preference for the soft EoS with compressibility $K=200 \mathrm{MeV}$ as used here in the IQMD calculations.

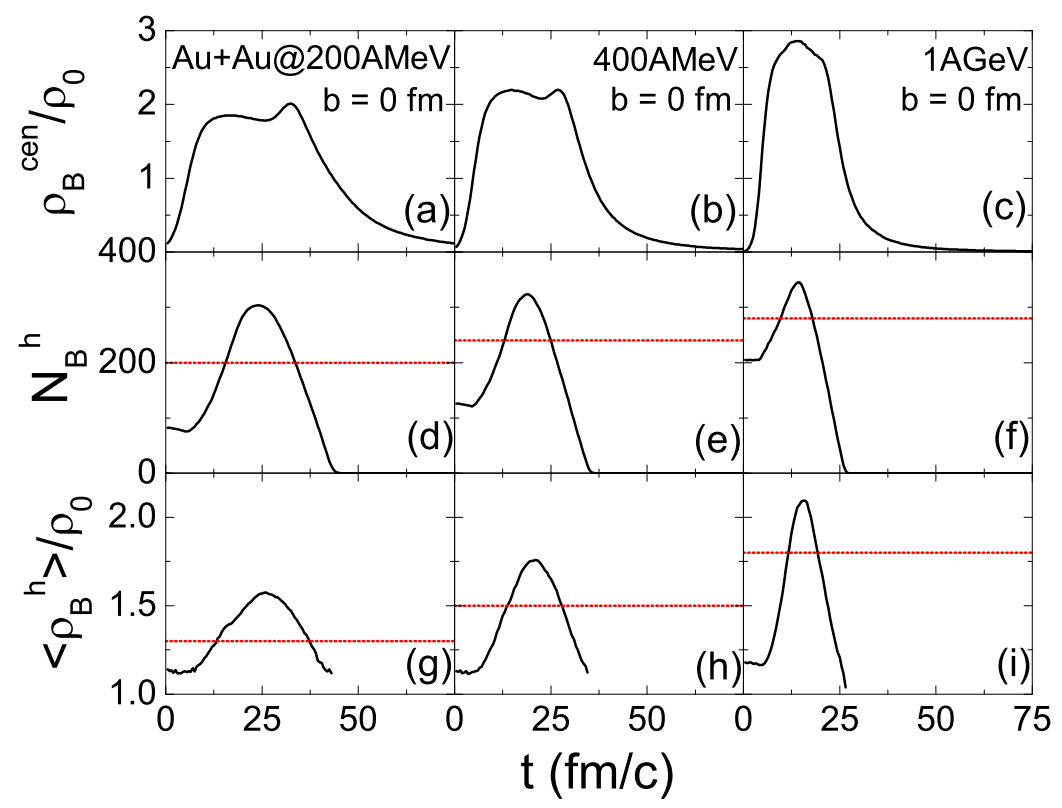

Figure 2: Temporal evolution of central baryon density (top), baryon multiplicity (middle), and average baryon density (bottom) in the high-density $\left(\rho>\rho_{0}\right)$ phase of central ${ }^{197} \mathrm{Au}+{ }^{197} \mathrm{Au}$ collisions at three energies ( $\rho_{0}$ is the saturation density). Horizontal lines indicate the average values used in the thermal model of Ref. [5] (reprinted with permission from Ref. [5]; Copyright (2013) by the American Physical Society).

The significance of collective flow and the sensitivity to the pressure and to the symmetric matter EoS has earlier been demonstrated by Danielewicz, Lacey and Lynch [3]. Parameterizations used in transport models were compared to state of the art many-body theories of the time. Extremely stiff and soft choices were ruled out on the basis of the experimental data for directed and elliptic flows for incident energies up to $10 \mathrm{GeV}$ per nucleon. The corresponding densities reached with heavy systems at these energies extend beyond four times the saturation value. The equation of state of asymmetric matter, the so-called asy-EoS, has also been addressed in this work and the additional effects of varying the assumptions on its stiffness are demonstrated.

The origin of collective motions is believed to reside in the initial high-density phase of the 
reaction $[3,4]$. According to transport theory, the central densities may reach twice the saturation value already at the moderate energy of $400 \mathrm{MeV}$ per nucleon at which the reaction dynamics is still dominated by hadronic degrees of freedom and mean-field effects. This is illustrated in Fig. 2 with results from isospin-dependent Boltzmann-Uehling-Uhlenbeck (IBUU) transport model calculations [5]. The high-density phase is short, typically $20 \mathrm{fm} / \mathrm{c}$, but involves a significant part of the colliding system.

Neutron stars are known to be built from nuclear matter with densities of similar and even higher magnitudes and high-density matter is also temporarily formed during the core-collapse phase of supernova explosions. Understanding and modeling these astrophysical phenomena requires the knowledge of nuclear matter properties far away from saturation and far away from symmetry. Besides the symmetric equation-of-state, also that of neutron-rich asymmetric matter has, therefore, received particular attention recently, especially also at high density at which its behavior is least well known $[6,7,8]$.

Earlier support for the softness of the symmetric-matter EoS has also been provided by the study of meson production ratios $[9,10]$. A soft EoS leads to higher densities, an increased rate of collisions and, therefore, higher kaon rates whose production preferentially proceeds through twostep processes. The usefulness of meson ratios for extracting information on the EoS of asymmetric matter or asy-EoS is, however, still not very clear. Four years ago, at the time of the XLVIII International Winter Meeting on Nuclear Physics in Memoriam of Ileana Iori, the situation was perhaps even confusing [11]. Probes considered as very promising as, e.g., $\mathrm{K}^{+} / \mathrm{K}^{0}$ production ratios had turned out to be only weakly sensitive to the density dependence of the symmetry energy when measured double ratios were compared to transport calculations $[12,13]$, and results deduced from pion ratios were found to be highly model dependent $[12,14,15]$. The $\pi^{-} / \pi^{+}$yield ratios measured by the FOPI Collaboration at several energies up to $1.5 \mathrm{GeV}$ per nucleon and for four mass-symmetric systems ${ }^{40} \mathrm{Ca}+{ }^{40} \mathrm{Ca},{ }^{96} \mathrm{Zr}+{ }^{96} \mathrm{Zr},{ }^{96} \mathrm{Ru}+{ }^{96} \mathrm{Ru}$, and ${ }^{197} \mathrm{Au}+{ }^{197} \mathrm{Au}[16]$ are still subject of intense studies within transport theory and the importance of in-medium effects has been emphasized [5, 17]. The theoretical analyses of this data set remain, however, ambiguous in their conclusions, suggesting everything from a rather stiff to a super-soft behavior of the symmetry energy $[14,15,18]$.

On the other hand, as shown two years ago at this conference [19], significant results regarding the high-density behavior of the asy-EoS have become available from the analysis of the isotopic dependence of collective flows [20,21]. Also here, the foremost question to be answered is that of potential model dependences of the obtained values. As for meson production, descriptions with transport models are required to extract the information of interest from the measured reaction data. Considerable attention has been given to this topic which will be in the center of the present report.

A comprehensive presentation of the broad experimental and theoretical, nuclear and astrophysical research devoted to the asymmetric-matter equation of state has very recently become available in the form of the "Topical issue on nuclear symmetry energy", a special volume of European Physical Journal A [22]. A brief introductory overview addressing the relevance of elliptic flow in the study of the symmetry energy at supra-saturation density may be found in Ref. [23]. 


\section{Collective flows}

The collective outflow of nuclear matter from the compressed interaction zone has first been observed in experiments at the Bevalac [4, 24]. In a sphericity analysis, the event shape in three dimensions was characterized by a kinetic-energy flow tensor whose main orientation with respect to the beam direction represents the collective sidewards flow and whose cross section, if it is non-isotropic, indicates the existence of elliptic flow. At the bombarding energies of up to 1.05 $\mathrm{GeV} /$ nucleon investigated in these studies, a preferential emission of charged particles perpendicular to the reaction plane has been observed. The shadowing by the spectator remnants as they pass each other during the collision reduces the in-plane emissions, so that the strength of the off-plane squeeze-out reflects the internal pressure and thus the effect of the equation of state. The squeezeout of neutrons has been first observed by the FOPI/LAND Collaboration [25, 26]. Their data is still available and has been used in the cited studies of the asy-EoS [20, 21].

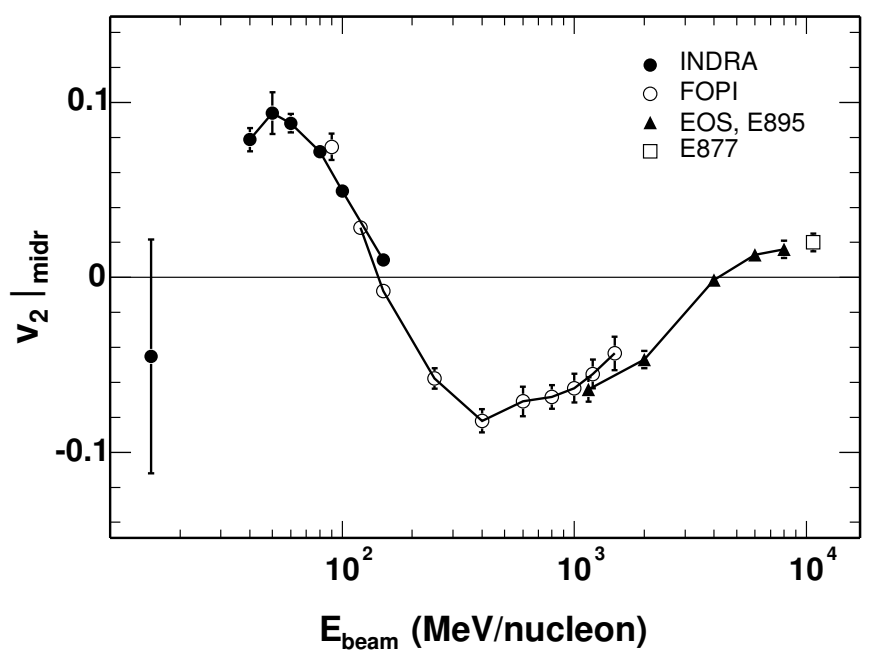

Figure 3: Elliptic flow parameter $v_{2}$ at mid-rapidity for ${ }^{197} \mathrm{Au}+{ }^{197} \mathrm{Au}$ collisions at intermediate impact parameters (about 5.5-7.5 fm) as a function of incident energy, in the beam frame. The filled and open circles represent the INDRA and FOPI data [32,33], respectively, for $Z=1$ particles, the triangles represent the EOS and E895 data [34] for protons and the square represents the E877 data [35] for all charged particles (from Ref. [31], reprinted with kind permission from Springer Science+Business Media).

Directed and elliptic flows, and if required also higher flow components, are commonly presented in the form of the coefficients obtained from a Fourier decomposition of the azimuthal distributions measured with respect to the orientation of the reaction plane $\phi_{R}[27,28,29]$,

$$
\frac{d N}{d\left(\phi-\phi_{R}\right)}=\frac{N_{0}}{2 \pi}\left(1+2 \sum_{n \geq 1} v_{n} \cos n\left(\phi-\phi_{R}\right)\right) .
$$

Here $N_{0}$ is the azimuthally integrated yield. The coefficients $v_{n} \equiv\left\langle\cos n\left(\phi-\phi_{R}\right)\right\rangle$ are functions of particle type, impact parameter, rapidity $y$, and the transverse momentum $p_{t}$.

Experimentally, the azimuthal angle of emission is determined with respect to the orientation of a reaction plane that has been reconstructed from observed emission patterns. Several methods 
have been proposed (see, e.g., Ref. [30, 31]) whose accuracy largely depends on the types and, in particular, on the multiplicities of particles detected in the reactions under study. The so-called dispersion of the reaction plane refers to the uncertainty of the reconstructed azimuthal orientation. The deduced coefficients are reduced in the case of poorly determined experimental reaction planes and corrections are necessary for which, however, quite refined methods exist. Their magnitude increases with the order of the Fourier coefficient considered.

An excitation function for the elliptic flow for $Z=1$ particles in ${ }^{197} \mathrm{Au}+{ }^{197} \mathrm{Au}$ collisions from various experiments is shown in Fig. 3. It extends up to the AGS regime of several GeV per nucleon incident energies at which in-plane flow starts to dominate again. Squeeze-out perpendicular to the reaction plane $\left(v_{2}<0\right)$, as a result of shadowing by the spectator remnants is observed at incident energies between about $150 \mathrm{MeV}$ per nucleon and $4 \mathrm{GeV}$ per nucleon with a maximum near $400 \mathrm{MeV}$ per nucleon. At lower energies, the collective rotation in the mean-field dominated dynamics causes the observed in-plane enhancement of emitted reaction products [36]. The figure also illustrates the precision that can be reached in flow measurements. The reliability of the applied methods is demonstrated by the good agreement of results from different experiments in the overlap regions of the studied intervals in collision energy [31, 32].

At even higher energies, in the ultrarelativistic regime, elliptic flow has become an increasingly important observable. The deduced constituent-quark scaling of elliptic flow is one of the prime arguments for deconfinement during the early collision phase, and properties of the formed quarkgluon liquid are obtained from the observed magnitude of collective motions [37, 38, 39, 40]. It implies that elliptic flow develops very early in the collision which is valid also in the present range of relativistic energies as confirmed by calculations [41]. Odd flow components, primarily the socalled triangular flow, are representative for the magnitude of initial state fluctuations [42] while the flow components of thermal photons as those of penetrating probes serve in studying the hot and dense medium created in ultra-relativistic heavy-ion collisions [43, 44].

At lower energies, the sensitivity of collective flows to essential features of the reaction dynamics is also continued to be exploited in various ways. In a systematic study with the updated ultrarelativistic quantum molecular dynamics (UrQMD) transport model, the sign and magnitude of directed flow were shown to depend crucially on several of the many parameters entering the calculation [45]. To correctly reproduce the magnitude of directed $Z=1$ flow observed for semicentral ${ }^{197} \mathrm{Au}+{ }^{197} \mathrm{Au}$ collisions between 40 and $150 \mathrm{MeV}$ per nucleon, a careful adjustment of the isoscalar EoS and of the density and momentum dependence of the in-medium nucleon-nucleon elastic cross sections had to be made. It emphasizes the need for consistent considerations of both, the mean field and the two-body collisions in transport models.

Dynamical flow observables, expected to be influenced by the asy-EoS in asymmetric systems, have been proposed by several groups as probes for the equation of state at high density $[46,47,48,49,50]$. The so-called differential neutron-proton flow is the difference of the parameters describing the collective motion of free neutrons and protons weighted by their numbers [48]. According to the simulations, this observable minimizes the influence of the isoscalar part in the EoS while maximizing that of the symmetry term [51]. Its proportionality to the particle multiplicities, however, makes its determination very dependent on the reliable distinction of free and bound nucleons in the experiment and in calculations. Therefore, mainly differences or ratios of the deduced flow coefficients have been considered up to now. 


\section{Symmetry energy and parameterization}

The symmetry energy, i.e. the symmetry-term coefficient $E_{\text {sym }}$ in the usual quadratic expansion of the equation of state,

$$
E / A(\rho, \delta)=E / A(\rho, \delta=0)+E_{\mathrm{sym}}(\rho) \cdot \delta^{2},
$$

is the difference between the energies of symmetric matter $(\delta=0)$ and neutron matter $(\delta=1)$ with $\delta=\left(\rho_{n}-\rho_{p}\right) / \rho$ and $\rho_{n}, \rho_{p}$, and $\rho$ representing the neutron, proton, and total densities, respectively.

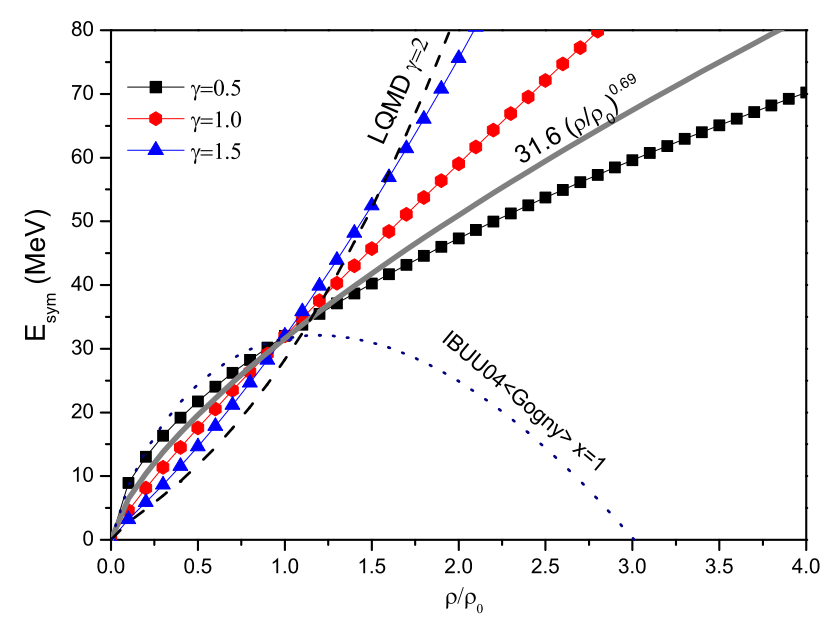

Figure 4: Parameterizations of the nuclear symmetry energy as used in transport codes: three parameterizations of the potential term used in the UrQMD (Ref. [52]) with power law coefficients $\gamma=0.5,1.0$, and 1.5 (lines with symbols as indicated), the result with $\gamma=0.69$ obtained from analyzing isospin diffusion data with the IBUU04 (full line, Ref. [54]), and the super-soft and stiff parameterizations obtained from analyzing the $\pi^{-} / \pi^{+}$production ratios with the IBUU04 (dotted line, Ref. [14]) and the ImIQMD (dashed line labeled LQMD, Ref. [15]) transport models (from Ref. [55], reprinted with kind permission from Springer Science+Business Media).

To be used in reaction models based on transport theory, often simplified descriptions of the composition-dependent part of the nuclear mean field are required. In the UrQMD [52, 53], in the version used for the analysis of the elliptic flow in ${ }^{197} \mathrm{Au}+{ }^{197} \mathrm{Au}[20]$, the density dependence of the potential part of the symmetry energy is described with the power-law coefficient $\gamma$, while a second parameter gives the strength at the saturation density $\rho_{0} \approx 0.16$ nucleons $/ \mathrm{fm}^{3}$,

$$
E_{\mathrm{sym}}=E_{\mathrm{sym}}^{\mathrm{pot}}+E_{\mathrm{sym}}^{\mathrm{kin}}=22 \mathrm{MeV} \cdot\left(\rho / \rho_{0}\right)^{\gamma}+12 \mathrm{MeV} \cdot\left(\rho / \rho_{0}\right)^{2 / 3},
$$

Examples of these parameterizations are given in Fig. 4. In the IBUU04 developed by the groups of $\mathrm{Li}$ and Chen $[6,54]$, the nuclear potential of Das et al. with explicit momentum dependence in the isoscalar and isovector parts is used [56]. The density dependence of the symmetry energy is characterized by a parameter $x$ appearing in the potential expressions. With $x=0$, the density dependence is similar to $\gamma=0.5$ and with $x=-1$ similar to $\gamma=1.3$, while $x=+1$ and $x=-2$ represent the super-soft and super-stiff cases, respectively. 
The density dependence of the symmetry energy is usually characterized in the form of the parameter $L$ which is proportional to the derivative with respect to density at saturation,

$$
L=3 \rho_{0} \cdot d E_{\mathrm{sym}} / d \rho \mid \rho_{0} .
$$

Quite compatible results, with their uncertainties falling into the interval $20 \mathrm{MeV} \leq L \leq 100 \mathrm{MeV}$, have been obtained over the past years from experiments testing the strength of the symmetry term at densities near or below saturation. This includes isospin diffusion and neutron/proton yield ratios [57] as well as the isospin dependence of the isoscalar giant monopole resonance [58] or the strength of the pygmy resonance in neutron rich nuclei [59]. The presently most favored value is $L \approx 60 \mathrm{MeV}$, roughly corresponding to a power-law coefficient $\gamma=0.6$ [60].

For very recent develoments, the reader is referred to the special volume of European Physical Journal A [22] and to the proceedings by Horowitz et al. reporting on a collaborative effort on improving our knowledge of the symmetry energy [61]. There, also the important role of the PREX experiment is emphasized which aims at measuring the parity-violating contribution to electron scattering at high energy [62]. It is considered a model-free determination of the neutron-skin thickness of ${ }^{208} \mathrm{~Pb}$, and possibly also other nuclei, which may serve as a benchmark for nuclearstructure studies of the same quantity $[63,64]$. Rather precise values for the symmetry energy and for the density to which the obtained results apply have been obtained from the analysis of isotope binding energy differences and the neutron-skin thicknesses of Sn isotopes [65] as well as from the properties of doubly-magic nuclei including the present value for the neutron skin thickness of ${ }^{208} \mathrm{~Pb}$ [66]. The densities are near $2 / 3$ of the saturation value which seems typical for the density regime that is probed with nuclear structure data and by studying isospin transport in fragmentation reactions.

On the theoretical side, highly constrained microscopic calculations have permitted rather precise predictions as, e.g., $L=66.5 \mathrm{MeV}$ for the slope parameter, obtained within the BruecknerHartree-Fock approach using the realistic Argonne V18 nucleon-nucleon potential and a phenomenological three-body force [67]. Three- and four-nucleon forces are included in the first complete $\mathrm{N}^{3} \mathrm{LO}$ calculations for neutron matter within chiral perturbation theory and shown to make significant contributions [68]. For the slope parameter, the interval $L=43-66.6 \mathrm{MeV}$ is obtained and expected to be narrowed further with future higher-order calculations. The density interval that has been covered with this approach reaches up to saturation, and extrapolations are required for reaching higher densities [69].

\section{Sensitivity to the symmetry energy}

The obvious need for probes of the strength of the symmetry energy at supra-saturation densities and the known connection of flow with early reaction phases has initiated broad studies of its sensitivity to the symmetry energy and searches for suitable observables. With UrQMD calculations, it was possible to demonstrate that the neutron and proton (or hydrogen) elliptic flows respond differently to variations of the strength of the symmetry-energy coefficient in the model [20]. Figure 5 shows the predictions for the elliptic-flow parameter $v_{2}$ of emitted neutrons, protons, and hydrogen isotopes for mid-peripheral ${ }^{197} \mathrm{Au}+{ }^{197} \mathrm{Au}$ collisions at $400 \mathrm{MeV}$ per nucleon and for the two choices of the density dependence of the symmetry energy, labeled asy-stiff $(\gamma=1.5)$ and 
asy-soft ( $\gamma=0.5$, cf. Fig. 4). They are displayed as a function of the laboratory rapidity $y_{\text {lab }}$. The dominant effect is the significantly larger neutron squeeze-out in the asy-stiff case as compared to the asy-soft case. The proton and hydrogen flows respond only weakly, and in opposite direction, to the variation of $\gamma$ within the chosen stiffness interval. Relative to each other, the neutron and proton elliptic flows vary on the level of $15 \%$. The absolute magnitude of the elliptic flow is also satisfactorily reproduced as shown by the comparison with the FOPI experimental result for $Z=1$ particles at mid-rapidity (Fig. 5). This has become possible through a proper choice made for the momentum dependence of the elastic nucleon-nucleon cross sections [45, 70]. In the present case, the FP1 parameterization has been chosen $[19,20]$.

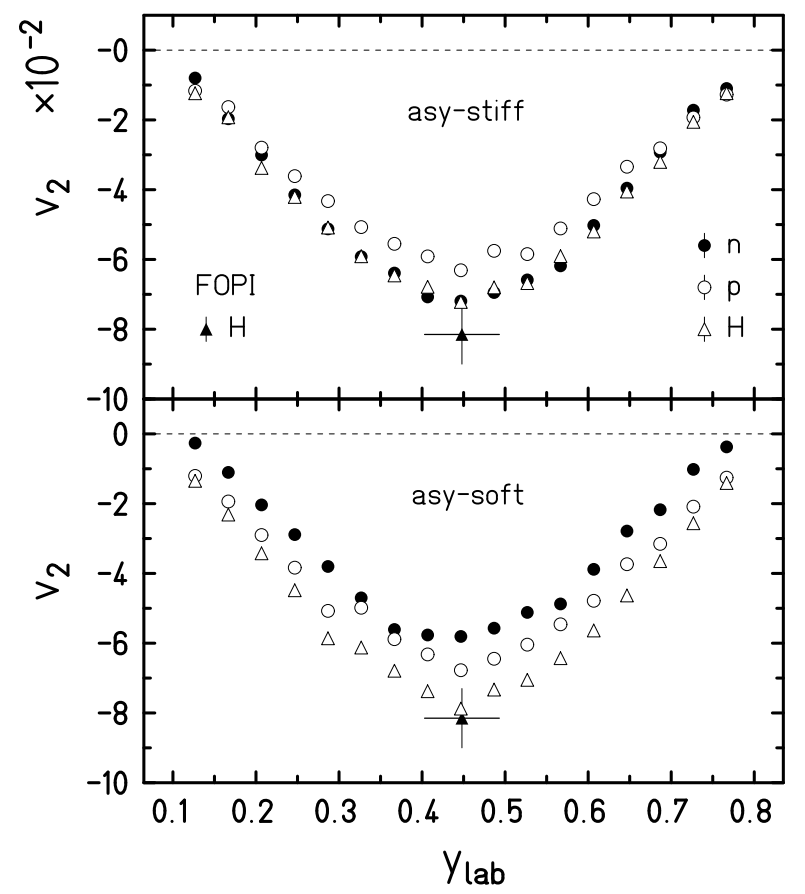

Figure 5: Elliptic flow parameter $v_{2}$ for mid-peripheral $(5.5 \leq b \leq 7.5 \mathrm{fm}){ }^{197} \mathrm{Au}+{ }^{197} \mathrm{Au}$ collisions at $400 \mathrm{MeV}$ per nucleon as calculated with the UrQMD model for neutrons (dots), protons (circles), and all hydrogen isotopes ( $Z=1$, open triangles), integrated over transverse momentum $p_{t}$, as a function of the laboratory rapidity $y_{\text {lab }}$. The predictions obtained with a stiff and a soft density dependence of the symmetry term are given in the upper and lower panels, respectively. The experimental result from Ref. [33] for $Z=1$ particles at mid-rapidity is represented by the filled triangle (reprinted from Ref. [20], Copyright (2011), with permission from Elsevier).

Following up on this first result, a more comprehensive study of the sensitivity of flow observables was performed within the newly updated version of the UrQMD model [71]. Calculations using 21 different Skyrme interactions, all exhibiting similar values of isoscalar incompressibility but very different density dependences of the symmetry energy were used to calculate predictions for the elliptic flow and its dependence on transverse momentum $p_{t}$ of free nucleons from ${ }^{197} \mathrm{Au}$ $+{ }^{197} \mathrm{Au}$ collisions at $400 \mathrm{MeV}$ per nucleon. Linear correlations were shown to exist between the slope parameter $L$ describing the density dependence of the symmetry energy and the differences, as well as the ratios, of the $p_{t}$-integrated elliptic flows of neutrons with respect to protons or hydrogen isotopes. 


\section{Elliptic flow from FOPI/LAND}

A data set for testing these predictions has been available from earlier experiments of the FOPI/LAND collaboration. It was originally collected and shown to provide evidence for the squeeze-out of neutrons emitted in ${ }^{197} \mathrm{Au}+{ }^{197} \mathrm{Au}$ collisions at $400 \mathrm{MeV}$ per nucleon [25]. The capability of the Large Area Neutron Detector LAND [72], used in these experiments of detecting neutrons as well as charged particles permitted the differential analysis of the observed flow patterns in the form of flow ratios [20] or flow differences [21], expected to enhance isovector with respect to isoscalar effects in the reaction dynamics.

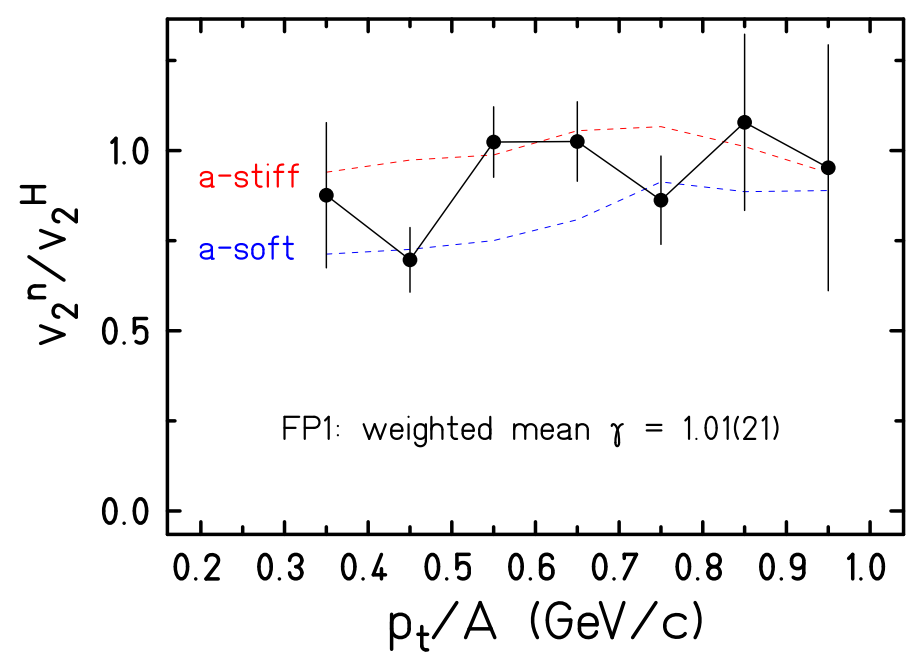

Figure 6: Ratio of the elliptic flow parameters $v_{2}$ for neutrons and hydrogen isotopes for moderately central $(b<7.5 \mathrm{fm})$ collisions of ${ }^{197} \mathrm{Au}+{ }^{197} \mathrm{Au}$ at $400 \mathrm{MeV}$ per nucleon as a function of the transverse momentum per nucleon $p_{t} / A$. The symbols represent the experimental data. The UrQMD predictions for $\gamma=1.5$ (a-stiff) and $\gamma=0.5$ (a-soft) obtained with the FP1 parameterization for elastic nucleon-nucleon cross sections [45] are given by the dashed lines (adapted from Ref. [20], Copyright (2011), with permission from Elsevier).

The measured ratio of neutron vs. hydrogen flows is shown in Fig. 6 as a function of the transverse momentum per nucleon $p_{t} / A$. The results of the UrQMD model for the two cases a-stiff $(\gamma=1.5)$ and a-soft $(\gamma=0.5)$ reflect the sensitivity to the density-dependent symmetry energy. The experimental ratios, even though associated with large errors, scatter within the interval given by the two calculations. A linear interpolation between the predictions, averaged over $0.3<p_{t} / A \leq 1.0$ $\mathrm{GeV} / \mathrm{c}$, yields $\gamma=1.01 \pm 0.21$ for the exponent describing the density dependence of the potential part of the symmetry energy (Eq. 3.2).

Particularly encouraging was the observation that the obtained result did not significantly depend on the choice made for the momentum dependence of the elastic nucleon-nucleon cross sections. With the FP2 parameterization [45], leading to nearly 40\% larger individual nucleon flows, the very similar value $\gamma=0.98 \pm 0.35$ was obtained. Only the error turned out to be larger as the predicted sensitivity was found to be slightly reduced in this case [20]. The observed insensitivity, nevertheless, supported the expectation that the role of isoscalar-type model ingredients is reduced for the differential flow observables.

After assessing statistical and systematic uncertainties, a value $\gamma=0.9 \pm 0.4$ was adopted by the authors, representing a moderately soft to linear density dependence of the symmetry energy. 
It falls slightly below the $\gamma=1.0$ line shown in Fig. 4 but, with the quoted uncertainty, stretches over a wide interval from $\gamma=0.5$ halfway up to $\gamma=1.5$. The corresponding slope parameter is $L=83 \pm 26 \mathrm{MeV}$. A comparable value $L=89 \pm 23 \mathrm{MeV}$ was deduced from the same data set in the more recent UrQMD study, performed with 21 Skyrme forces [71].

In an independent analysis, Cozma has used data from the same experiment, however sorted slightly differently [26], and investigated the influence of several parameters on the difference between the elliptic flows of protons and neutrons using the Tübingen version of the QMD transport model [21]. A super-soft behavior of the symmetry energy was confirmed to be excluded by the comparison with the experimental flow data. Also there, the reduced significance of model parameters affecting neutron and proton dynamics in similar ways was demonstrated. In particular, the dependence of the elliptic-flow difference on the choice made for the symmetry energy was shown to considerably exceed that made for the symmetric-matter equation of state. This work was continued, leading to a very systematic study of the model dependence of the determined properties of the symmetry energy [73].

\section{Study of model dependence}

An investigation of the overall parameter dependence has been performed with the Tübingen version of the QMD transport model [73]. The effects of the selected microscopic nucleon-nucleon cross-sections, of the compressibility of nuclear matter, of the optical potential, and of the parameterization of the nucleon wave packets were thoroughly studied. For the symmetry energy, the parameterization derived from the momentum dependent interaction (MDI) of Das et al. [6, 54, 56] and a power-law parameterization were both used and the results compared with the experimental FOPI/LAND data for ${ }^{197} \mathrm{Au}+{ }^{197} \mathrm{Au}$ collisions at $400 \mathrm{MeV}$ per nucleon. The acceptance cuts of the experiment were applied.

The chosen procedure consisted in starting with a set of model parameters that leads to a satisfactory reproduction of the individual nucleon flows (within 15\%) and of the neutron-proton flow difference and ratio for a given choice of the asymmetry parameter $x$ of the MDI interaction. Other parameters were then varied within the commonly accepted limits found in the literature. This is illustrated in Fig. 7 which shows the error bands arising from the individual uncertainties and their quadratically added sum in comparison with the experimental result for the neutron-proton elliptic-flow difference. The central theoretical results (full and dashed lines) were obtained with two parameterizations of the nucleon-nucleon cross sections, labelled Cugnon and Li-Machleidt [75, 76], with a soft symmetric-matter EoS $K=210 \mathrm{MeV}$ and a width of the nucleon wave function $L=4.33 \mathrm{fm}^{2}$. For the optical potential, the version of Hartnack and Aichelin [77] was used.

The figure shows that the effects of choosing different cross section parameterizations had almost an order-of-magnitude smaller effect on the elliptic-flow difference than variations of the density dependence of the symmetry energy through changing $x$. A large part of the total uncertainty is contributed by the symmetric-matter compressibility modulus which has been varied within the range $K=190-300 \mathrm{MeV}$. Altogether, the global variation of the general model parameters within commonly accepted limits leads to finite uncertainties of typically one unit of the $x$ parameter describing the stiffness of the symmetry energy in the MDI parameterization. The cor- 


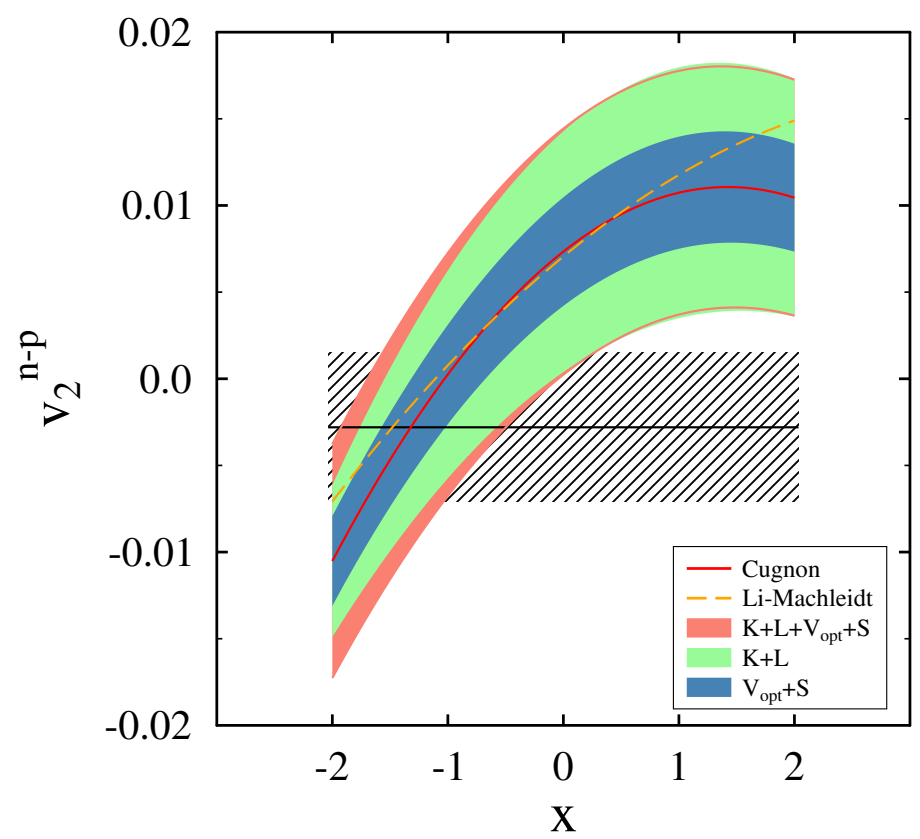

Figure 7: Model dependence of the neutron-proton elliptic-flow difference in comparison with FOPI/LAND experimental data, integrated over impact parameter $b<7.5 \mathrm{fm}$, normalized rapidity $0.25<y / y_{p}<0.75$, and transverse momentum $0.3<p_{t}<1.0 \mathrm{GeV} / \mathrm{c}$, shown as a function of the MDI model parameter $x$. The sensitivities to the compressibility modulus $(K)$, the width of the nucleon wave function $(L)$, the optical potential $\left(V_{\mathrm{opt}}\right)$, and the parameterization of the symmetry energy $(S)$ are displayed. The total model dependence is obtained by adding in quadrature individual sensitivities. (from Ref. [74], reprinted with kind permission from Springer Science+Business Media).

responding value of the slope parameter is $L \approx 120 \pm 55 \mathrm{MeV}$ where the error includes the overall systematic model dependence as determined in this study [73].

In more detail, the effects of choosing different cross section parameterizations had almost an order-of-magnitude smaller effect on the difference between proton and neutron elliptic flows than variations of the density dependence of the symmetry energy. This is consistent with the very small influence of the momentum-dependence of the nucleon-nucleon cross sections in the UrQMD study discussed in the previous section. On the other hand, the interval chosen for the isoscalar compressibility can be considered larger than what is compatible with recent experimental results (cf. Fig. 1) and, furthermore, gave rise to departures of the individual $v_{2}$ from the experimental values by as much as $40-50 \%$. This may be seen as very encouraging as a further reduction of the theoretical uncertainties together with more precise experimental data may allow us to reach narrower constraints for the symmetry energy in the density regime probed with collective flows. This includes the role of the momentum dependence of the asy-Eos which has been addressed by using momentum-dependent and independent parameterizations and by adding the observed effects into the overall uncertainty [73].

Figure 8 shows explicitly the constraint on the density dependence of the symmetry energy obtained from this comprehensive study of the overall model dependence, performed in comparison with the experimental results for the nucleon elliptic flow in ${ }^{197} \mathrm{Au}+{ }^{197} \mathrm{Au}$ collisions at 400 $\mathrm{MeV}$ per nucleon. For comparison, the earlier UrQMD result [20] has been added. The two stud- 


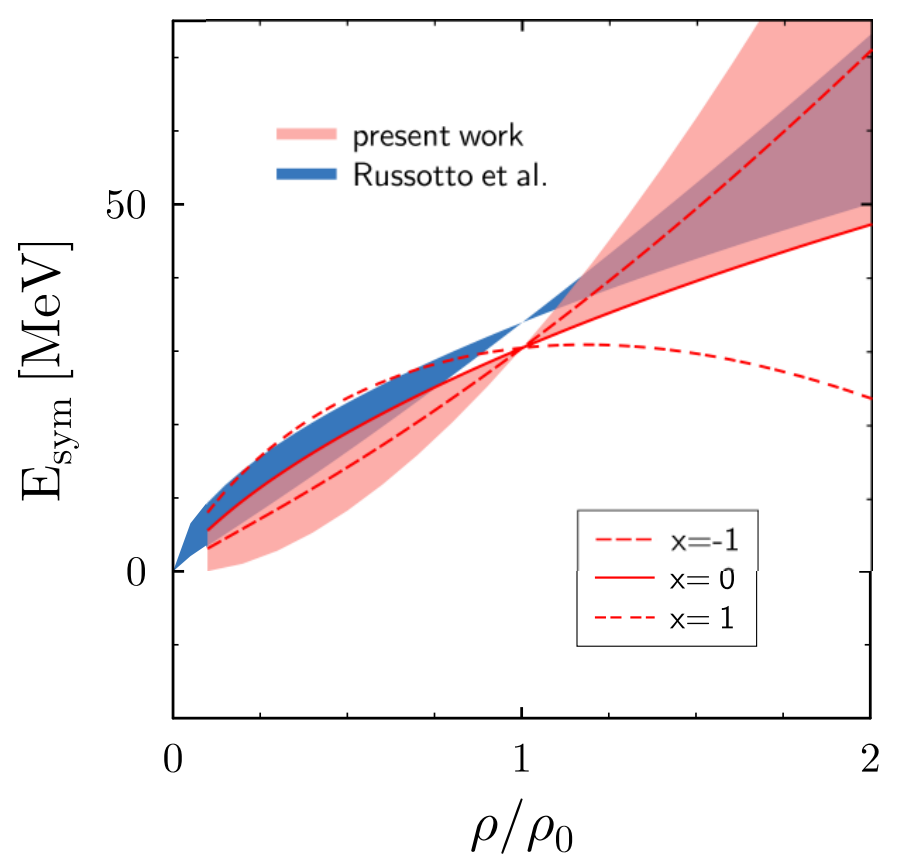

Figure 8: Constraints on the density dependence of the symmetry energy obtained from comparing theoretical predictions for the neutron-proton elliptic-flow difference and ratio to FOPI/LAND experimental data (labelled present work, Ref. [73]). The result of Ref. [20] is also shown together with the MDI parameterization of the symmetry energy for three values of the stiffness parameter: $x=-1$ (stiff), $x=0$ and $x=1$ (soft) (reprinted with permission from Ref. [73]; Copyright (2013) by the American Physical Society).

ies employ independent flavors of the QMD transport model (Tübingen QMD vs. UrQMD) and parameterizations of both, the isoscalar and isovector EoS that differ. The constraints on the density dependence of the symmetry energy obtained with these different ingredients are in agreement with each other which contrasts with the current status of the effort to constrain the symmetry energy from $\pi^{-} / \pi^{+}$ratios. By combining the two estimates, a moderately stiff to linear density dependence corresponding to a parameterization $x=-1.0 \pm 1.0$ has been obtained. It indicates a somewhat faster increase of the symmetry energy with density than what is extracted from nuclear structure and reactions for sub-saturation densities.

\section{Present status of ASY-EOS}

The ASY-EOS experiment, conducted at the GSI laboratory in 2011, represents an attempt to considerably improve the statistical accuracy of the flow parameters for the ${ }^{197} \mathrm{Au}+{ }^{197} \mathrm{Au}$ system at $400 \mathrm{MeV}$ per nucleon but also to complement these measurements with other observables and data for other systems [78]. Additional constraints for the comparison with transport models were considered to be useful for narrowing down the uncertainties of their predictions as shown in the previous section. For this purpose, data were collected for two additional systems, the neutronrich ${ }^{96} \mathrm{Zr}+{ }^{96} \mathrm{Zr}$ and neutron-poor ${ }^{96} \mathrm{Ru}+{ }^{96} \mathrm{Ru}$ pair of mass-symmetric $A=96$ collision systems, both also at $400 \mathrm{MeV}$ per nucleon incident energy. The FOPI Collaboration has studied these three reactions in quite some detail, at the present energy $400 \mathrm{MeV}$ per nucleon but also at other bombarding energies up to $1.5 \mathrm{GeV}$ per nucleon [1]. 
A schematic view of the experimental set-up is shown in Fig. 9. The beam was guided in vacuum to about $2 \mathrm{~m}$ upstream from the target. A thin plastic foil viewed by two photo-multipliers was used to record the projectile arrival times and to serve as a start detector for the time-of-flight measurement. The Large Area Neutron Detector (LAND, Ref. [72]), recently upgraded with new TACQUILA GSI-ASIC electronics, was positioned at a laboratory angle near $45^{\circ}$ with respect to the beam direction at a distance of about $5 \mathrm{~m}$ from the target. Its kinematical acceptance was similar to that of the forward LAND subdetector used in the FOPI/LAND experiment [20] but slightly larger in rapidity for given transverse momentum due to the shorter distance from the target. The veto-wall of plastic scintillators in front of LAND permitted the distinction between neutral and charged particles.

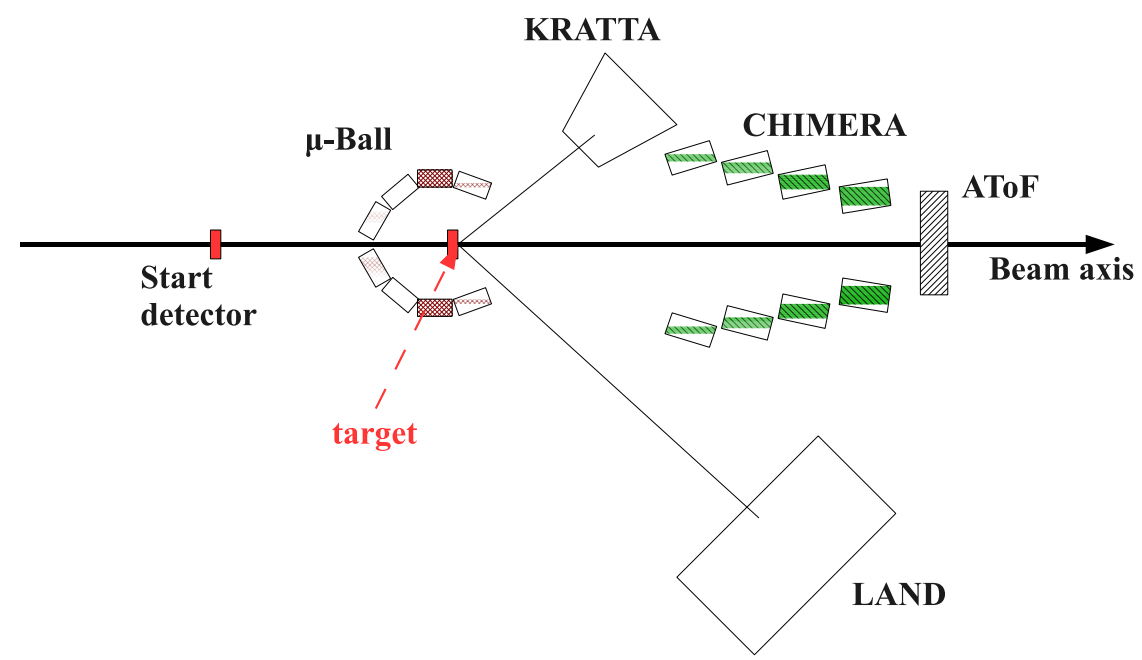

Figure 9: Schematic view of the experimental setup used in the ASY-EOS experiment S394 at GSI showing the six main detector systems and their positions relative to the beam direction. The dimensions of the symbol and the distances are not to scale (from Ref. [79]).

Opposite of LAND, covering a comparable range of polar angles, the Kraków Triple Telescope Array KraTTA [80] had been installed in order to improve the capabilities for measuring charged particle flows under the same experimental conditions. KraTTA had been specifically designed for the experiment to measure the energy, emission angles, and isotopic composition of light charged reaction products. Its 35 individual triple telescopes, arranged in a 7x5 array, subtended together a solid angle of $160 \mathrm{msr}$ with respect to the target.

For the event characterization and for measuring the orientation of the reaction plane, three detection systems had been installed. The ALADIN Time-of-Flight wall [81] was used to detect charged particles and fragments in forward direction at polar angles up to $\theta_{\mathrm{lab}} \leq 7^{\circ}$. Its capability of identifying large fragments and of providing the input for the sorting variable $Z_{\text {bound }}$ [81] permitted the event sorting according to impact parameter. Four double rings of the CHIMERA multidetector [82] carrying together $352 \mathrm{CsI}(\mathrm{Tl})$ scintillators in forward direction and four rings with 50 thin $\mathrm{CsI}(\mathrm{Tl})$ elements of the Washington-University $\mu$-ball array [83] surrounding the target provided sufficient coverage and granularity for determining the orientation of the reaction plane from the measured azimuthal particle distributions. The kinematic coverage achieved with this assembly of detection systems is illustrated in Fig. 10. 


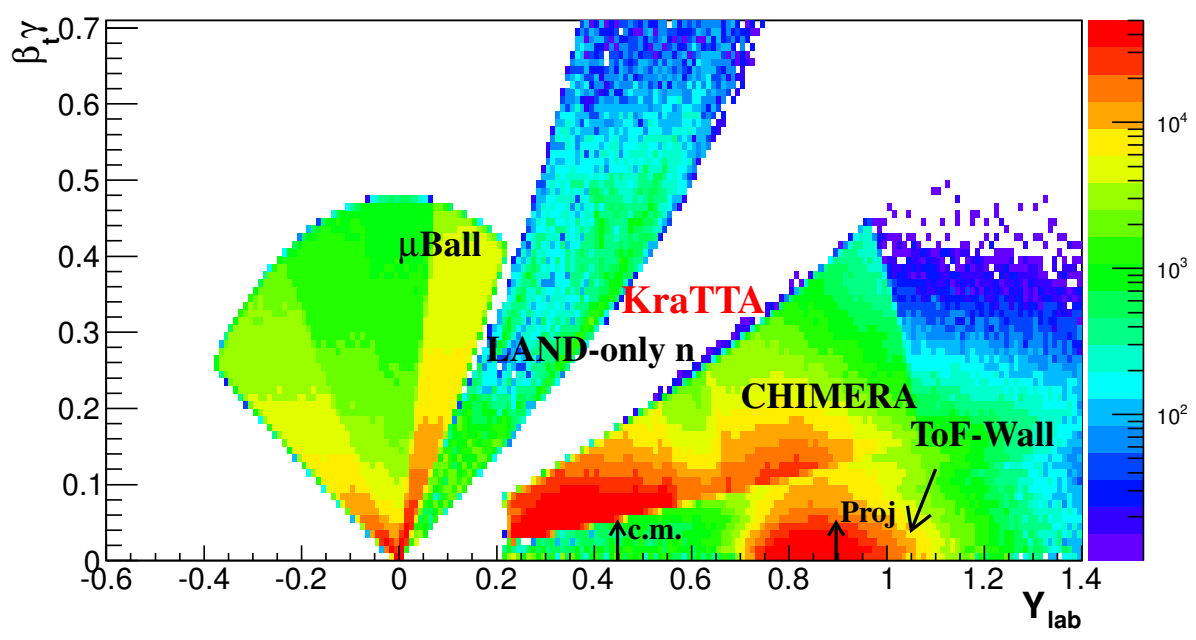

Figure 10: Invariant hit distribution in the $\gamma \beta_{t}$ vs. rapidity $y_{\text {lab }}$ plane for charged particles detected with the three systems $\mu$-Ball, CHIMERA, and ToF-Wall with full azimuthal coverage and neutrons detected with LAND. The acceptance of KRATTA is similar to that of LAND (from Ref. [79]).

Preliminary results from the still ongoing analysis have been presented at conferences. A highly improved statistical accuracy has been achieved and it has become evident that the strong fluctuations observed in the FOPI/LAND data as a function of the transverse momentum $p_{t} / A$ (Fig. 6) are of primarily statistical origin. At the time of the present conference, a value of $\gamma=$ $0.84 \pm 0.11$ (statistical) had become available from the analysis of neutron and charged-particle flows within the interval $0.3 \leq p_{t} \leq 0.7$ from ${ }^{197} \mathrm{Au}+{ }^{197} \mathrm{Au}$ collisions at $400 \mathrm{MeV}$ per nucleon and from the comparison of the flow ratios with UrQMD calculations adapted to the conditions of the experiment [84]. It corresponds to a slope parameter $L=79 \pm 8 \mathrm{MeV}$ which, within errors, confirms the density dependence of the symmetry energy obtained with FOPI/LAND. This result is, however, preliminary with regard to specific systematic uncertainties that at present are still under study.

\section{Summary and Outlook}

The performed studies of the model dependence are important steps towards establishing the elliptic flows of nucleons and light charged particles in neutron-rich collision systems as a source of information on the equation of state of asymmetric matter. By using different parameterizations of the optical potential and of the symmetry energy and by varying the remaining parameters within commonly accepted limits, it has been possible to show that the overall model dependence causes uncertainties not larger than those of the presently existing experimental data. With both, additional experimental and theoretical work, narrower constraints will be within reach. Adopting a soft solution with closer limits for the isoscalar compressibility, as suggested by the recent FOPI data [1], can be a possible first step. The completion of the ASY-EOS data analysis is an important task for the very near future.

The analysis of the existing FOPI/LAND data for ${ }^{197} \mathrm{Au}+{ }^{197} \mathrm{Au}$ collisions at $400 \mathrm{MeV}$ per nucleon with the UrQMD and Tübingen QMD transport models has led to constraints between a 
moderately soft to moderately stiff density dependence of the symmetry energy with corresponding slope parameters $L \approx 85 \pm 25 \mathrm{MeV}$, as obtained with the $\operatorname{UrQMD}[20,71]$, and the value $L \approx 120 \pm$ $55 \mathrm{MeV}$ obtained with the Tübingen QMD whose error represents the overall model dependence determined in this study [73]. Extreme solutions, super-soft or super-stiff, are not compatible with these results.

Future experiments with reaction systems of larger asymmetry will be feasible and important. Radioactive beam facilities will provide new possibilities for using neutron-rich beams, preferably combined with isotopically enriched targets. More efficient detector setups will be needed to compensate the corresponding loss in luminosity. The neutron detector NeuLAND [85] presently under construction for experiments at FAIR will offer a highly improved detection efficiency in the lower range of energies and improved calorimetric properties, thus permitting the simultaneous measurement of neutrons and light charged particles with high resolution.

The continuing fruitful collaboration and illuminating discussions with M.D. Cozma, A. Le Fèvre, Y. Leifels, R. Lemmon, Q. Li, J. Łukasik, W. Reisdorf, P. Russsotto, and H.H. Wolter are gratefully acknowledged.

\section{References}

[1] W. Reisdorf et al., Nucl. Phys. A 876 (2012) 1.

[2] C. Hartnack et al., Eur. Phys. J. A 1 (1998) 151.

[3] P. Danielewicz, R. Lacey, W.G. Lynch, Science 298 (2002) 1592.

[4] H.H. Gutbrod, A.M. Poskanzer, H.G. Ritter, Rep. Prog. Phys. 52 (1989) 1267.

[5] Jun Xu, Lie-Wen Chen, Che Ming Ko, Bao-An Li, Yu-Gang Ma, Phys. Rev. C 87 (2013) 067601.

[6] for a review, see Bao-An Li, Lie-Wen Chen, and Che Ming Ko, Phys. Rep. 464 (2008) 113.

[7] M. Di Toro, V. Baran, M. Colonna, V. Greco, J. Phys. G 37 (2010) 083101.

[8] C. Fuchs and H.H. Wolter, Eur. Phys. J. A 30 (2006) 5.

[9] C. Sturm et al., Phys. Rev. Lett. 86 (2001) 39.

[10] C. Fuchs, A. Faessler, E. Zabrodin, Y.M. Zheng, Phys. Rev. Lett. 86 (2001) 1974.

[11] W. Trautmann, in proceedings of XLVIII International Winter Meeting on Nuclear Physics in Memoriam of Ileana Iori, POS (BORMI02010) 061.

[12] G. Ferini et al., Nucl. Phys. A 762 (2005) 147; Phys. Rev. Lett. 97 (2006) 202301.

[13] X. Lopez et al., Phys. Rev. C 75 (2007) 011901(R).

[14] Zhigang Xiao et al., Phys. Rev. Lett. 102 (2009) 062502.

[15] Zhao-Qing Feng and Gen-Ming Jin, Phys. Lett. B 683 (2010) 140.

[16] W. Reisdorf et al., Nucl. Phys. A 781 (2007) 459.

[17] Hermann Wolter, in proceedings of $50^{\text {th }}$ International Winter Meeting on Nuclear Physics, POS (BORMIO2012) 059.

[18] W.-J. Xie, J. Su, L. Zhu, F.-S. Zhang, Phys. Lett. B 718 (2013) 1510.

[19] W. Trautmann and QingFeng $\mathrm{Li}$, in proceedings of $50^{\text {th }}$ International Winter Meeting on Nuclear Physics, POS (BORMIO2012) 060.

[20] P. Russotto et al., Phys. Lett. B 697 (2011) 471.

[21] M.D. Cozma, Phys. Lett. B 700 (2011) 139.

[22] Topical issue on nuclear symmetry energy, edited by Bao-An Li, Àngels Ramos), Giuseppe Verde, and Isaac Vidaña, Eur. Phys. J. A $\mathbf{5 0}$ (2014).

[23] W. Trautmann and H.H. Wolter, Int. J. Mod. Phys. E 21 (2012) 1230003. 
[24] H.H. Gutbrod et al., Phys. Rev. C 42 (1990) 640.

[25] Y. Leifels et al., Phys. Rev. Lett. 71 (1993) 963.

[26] D. Lambrecht et al., Z. Phys. A 350 (1994) 115.

[27] S. Voloshin and Y. Zhang, Z. Phys. C 70 (1996) 665.

[28] J.-Y. Ollitrault, preprint arXiv:nucl-ex/9711003 (1997).

[29] A.M. Poskanzer and S.A. Voloshin, Phys. Rev. C 58 (1998) 1671.

[30] P. Danielewicz and G. Odyniec, Phys. Lett. B 157 (1985) 146.

[31] A. Andronic, J. Łukasik, W. Reisdorf, W. Trautmann, Eur. Phys. J. A 30 (2006) 31.

[32] J. Łukasik et al., Phys. Lett. B 608 (2005) 223.

[33] A. Andronic et al., Phys. Lett. B 612 (2005) 173.

[34] C. Pinkenburg et al., Phys. Rev. Lett. 83 (1999) 1295.

[35] P. Braun-Munzinger and J. Stachel, Nucl. Phys. A 638 (1998) 3c.

[36] M.B. Tsang et al., Phys. Rev. C 47 (1993) 2717.

[37] B.I. Abelev et al. (STAR Collaboration), Phys. Rev. Lett. 99 (2007) 112301.

[38] K. Aamodt et al. (ALICE Collaboration), Phys. Rev. Lett. 105 (2010) 252302.

[39] A. Adare et al. (PHENIX Collaboration), Phys. Rev. C 85 (2012) 064914.

[40] G. Aad et al. (ATLAS Collaboration), Phys. Lett. B 707 (2012) 330.

[41] P. Danielewicz, Nucl. Phys. A 673 (2000) 375.

[42] A. Adare et al. (PHENIX Collaboration), preprint, arXiv:1401.7680[nucl-ex] (2014).

[43] C. Shen, U. Heinz, J.-F. Paquet, C. Gale, preprint, arXiv:1403.7558[nucl-th] (2013).

[44] G. Vujanovic et al., preprint, arXiv:1404.3714[hep-ph] (2014).

[45] Qingfeng Li et al., Phys. Rev. C 83 (2011) 044617.

[46] L. Scalone, M. Colonna, M. Di Toro, Phys. Lett. B 461 (1999) 9.

[47] Bao-An Li, A.T. Sustich, Bin Zhang, Phys. Rev. C 64 (2001) 054604.

[48] Bao-An Li, Phys. Rev. Lett. 88 (2002) 192701.

[49] V. Greco et al., Phys. Lett. B 562 (2003) 215.

[50] V. Baran, M. Colonna, V. Greco, M. Di Toro, Phys. Rep. 410 (2005) 335.

[51] Gao-Chan Yong, Bao-An Li, Lie-Wen Chen, Phys. Rev. C 74 (2006) 064617.

[52] Q. Li et al., J. Phys. G 31 (2005) 1359.

[53] Q. Li et al., J. Phys. G 32 (2006) 151; ibid. 32 (2006) 407.

[54] Bao-An Li and Lie-Wen Chen, Phys. Rev. C 72 (2005) 064611.

[55] ChenChen Guo et al., Science China Physics, Mechanics \& Astronomy 55 (2012) 252.

[56] C.B. Das, S. Das Gupta, C. Gale, Bao-An Li, Phys. Rev. C 67 (2003) 034611.

[57] M.B. Tsang et al., Phys. Rev. Lett. 102 (2009) 122701.

[58] T. Li et al., Phys. Rev. C 81 (2010) 034309.

[59] A. Klimkiewicz et al., Phys. Rev. C 76 (2007) 051603(R).

[60] Bao-An Li and Xiao Han, preprint, arXiv:1304.3368[nucl-th] (2013).

[61] C.J. Horowitz et al., preprint, arXiv:1401.5839[nucl-th] (2014).

[62] S. Abrahamyan et al., Phys. Rev. Lett. 108 (2012) 112502.

[63] C.J. Horowitz et al., Phys. Rev. C 85 (2012) 032501(R).

[64] K. Kumar, in proceedings of $50^{\text {th }}$ International Winter Meeting on Nuclear Physics, POS (BORMIO2012) 042.

[65] Zhen Zhang and Lie-Wen Chen, preprint arXiv:1302.5327[nucl-th] (2013).

[66] B. Alex Brown, preprint arXiv:1308.3664[nucl-th] (2013).

[67] I. Vidanña, C. Providência, A. Polls, A. Rios, Phys. Rev. C 80 (2009) 045806. 
[68] I. Tews, T. Krüger, K. Hebeler, A. Schwenk, Phys. Rev. Lett 110 (2013) 032504.

[69] K. Hebeler, J.M. Lattimer, C.J. Pethick, A. Schwenk, Phys. Rev. Lett. 105 (2010) 161102.

[70] Y. Wang, C. Guo, Q. Li, H. Zhang, Z. Li, W. Trautmann, Phys. Rev. C 89 (2014) 034608.

[71] Y. Wang, C. Guo, Q. Li, H. Zhang, Y. Leifels, W. Trautmann, Phys. Rev. C 89 (2014) 044603.

[72] Th. Blaich et al., Nucl. Instrum. Methods Phys. Res. A 314 (1992) 136.

[73] M.D. Cozma, Y. Leifels, W. Trautmann, Q. Li, P. Russotto, Phys. Rev. C 88 (2013) 044912.

[74] P. Russotto et al., Eur. Phys. J. A 50:38 (2014).

[75] G.Q. Li and R. Machleidt, Phys. Rev. C 48 (1993) 1702.

[76] G.Q. Li and R. Machleidt, Phys. Rev. C 49 (1994) 566.

[77] C. Hartnack and J. Aichelin, Phys. Rev. C 49 (1994) 2801.

[78] P. Russotto et al., in Proceedings of the $11^{\text {th }}$ International Conference on Nucleus-Nucleus Collisions, San Antonio, Texas, USA, 2012, edited by Bao-An Li and J.B. Natowitz, J. Phys. Conf. Ser. 420 (2013) 012092; preprint, arXiv:1209.5961[nucl-ex] (2012).

[79] P. Russotto et al., in Proceedings of the International Nuclear Physics Conference INPC2013, Firenze, Italy, 2013, EPJ Web of Conferences 66 (2014) 03074.

[80] J. Łukasik et al., Nucl. Instrum. Methods Phys. Res. A 709 (2013) 120.

[81] A. Schüttauf et al., Nucl. Phys. A 607 (1996) 457.

[82] A. Pagano et al., Nucl. Phys. A 734 (2004) 504.

[83] D.G. Sarantites et al., Nucl. Instr. and Meth. A 381 (1996) 418.

[84] P. Russotto and ASY-EOS Collaboration, work in progress (2014).

[85] NeuLAND Technical Design Report, submitted to FAIR (2011). 